\title{
Características dos atendimentos às vítimas de trauma admitidas em um pronto socorro via transporte aéreo
}

\author{
Characteristics of assistance to trauma victims admitted to an emergency room via air medical \\ transport
}

\section{Características de la atención a víctimas de traumas admitidos en el servicio de urgencias vía transporte aéreo}

\begin{abstract}
RESUMO
Objetivo: Descrever as características dos atendimentos às vítimas de trauma admitidas em um pronto socorro de grande porte, via transporte aéreo. Método: Estudo transversal que analisou 107 prontuários de vítimas de trauma admitidas via transporte aéreo. Os dados foram submetidos à análise estatística descritiva. Resultados: Predominou o sexo masculino (63,3\%), mediana de idade de 32 anos (IQ:23-51), vítimas de colisão automobilística $(28,0 \%)$, transportadas por serviço aéreo público $(86,0 \%)$ e nível de prioridade vermelho (55,7\%). À admissão, 55,0\% possuíam prótese de vias aéreas e $57,9 \%$ tiveram lesão na região do crânio. $72,9 \%$ realizou tomografia, $60,7 \%$ recebeu tratamento cirúrgico e $12,1 \%$ evoluiu a óbito no pronto socorro. O tempo de internação hospitalar teve mediana de sete dias (IQ: 1,5 -33,0), 57,0\% recebeu alta domiciliar e 26,2\% evoluiu a óbito. Conclusão: As vítimas, maioritariamente, eram graves e demandaram atendimento imediato e especializado, incluindo propedêuticas e terapêuticas de alta complexidade.

Descritores: Cuidados de Suporte Avançado de Vida no Trauma; Resgate Aéreo; Serviços Médicos de Emergência; Enfermagem em Emergência.
\end{abstract}

\begin{abstract}
Objective: To describe the characteristics of care provided to trauma victims admitted to an emergency room (ER) by air medical transport. Method: 107 medical records were collected and submitted to descriptive statistical analysis. Results: Prevalence of male individuals (63.3\%), at a median age of 32 years (IQR: 23-51), car crash as a trauma mechanism (28.0\%), public air transport (86.0\%), and emergency priority level (55.7\%). Upon admission, $55.0 \%$ had airway prosthesis, $68.2 \%$ received supplemental oxygen and $85.0 \%$ were immobilized on a long backboard. Limbs (66.3\%) and skull (57.9\%) were the most affected body regions. Tomography was performed in $72.9 \%$. $60.7 \%$ received surgical treatment while $12.1 \%$ died in the ER. Length of hospital stay was seven days (IQR: 1.5 -33.0). More than half were discharged (57.0\%) and $26.2 \%$ died. Conclusion: Trauma victims admitted via air medical transport were mostly in serious condition and demanded immediate, specialized care, including highly complex care.

Descriptors: Advanced Trauma Life Support Care; Air Ambulances; Emergency Medical Services; Emergency Nursing.
\end{abstract}

\section{RESUMEN}

Objetivo: Describir las características de la atención a las víctimas de trauma admitidas en un servicio de urgencias vía transporte aéreo. Método: Este estudio transversal analizó 107 historias clínicas de pacientes víctimas de trauma, admitidos en un servicio de urgencias vía transporte aéreo. Resultados: Predominó el sexo masculino (63,3\%), edad mediana de 32 años (IQ:23-51), colisión automovilística como mecanismo de lesión $(28,0 \%)$, transporte por servicio aéreo público $(86,0 \%)$ y nivel de prioridad emergencia (55,7\%). En la admisión, 55,0\% tenían prótesis de vía aérea, 68,2\% recibian oxígeno suplementario y $85,0 \%$ estaban inmovilizados en tablas largas. Los miembros $(66,3 \%)$ y cráneo $(57,9 \%)$ fueron las regiones corporales más afectadas. La tomografía fue realizada en $72,9 \%, 60,7 \%$ recibió tratamiento quirúrgico y $12,1 \%$ evolucionaron a óbito. El tiempo de internación fue de siete días (IQ: 1,5-33,0). 57,0\% recibió alta domiciliaria y 26,2\% evolucionaron a óbito. Conclusiones: Las víctimas de trauma admitidas vía transporte aéreo eran en su mayoría graves y demandaron atención inmediata y especializada, incluyendo propedéuticas y terapéuticas de alta complejidad.

Descriptores: Atención de Apoyo Vital Avanzado en Trauma; Ambulancias Aéreas; Servicios Médicos de Urgencia; Enfermería de Urgencia.
Caio Henrique Macedo Camargos de Oliveira ${ }^{1}$

0000-0001-9423-5190

Thaís Regina Gomes da Silva ${ }^{1}$ (D) 0000-0002-6869-9634

Thaís Moreira Oliveira ${ }^{1}$

0000-0001-6481-7608

Frederico Bruzzi de Carvalho² (D) 0000-0002-9752-5625

Allana dos Reis Corrêa ${ }^{1}$ (iD) 0000-0003-2208-958X

1 Universidade Federal de Minas Gerais.

2 Fundação Hospitalar do Estado de Minas Gerais.

Autor correspondente:

Caio Henrique Macedo Camargos de

Oliveira

E-mail: enfcaiohmacedo@gmail.com 


\section{INTRODUÇÃO}

Na Classificação Internacional de Doenças (CID-10), definem-se causas externas como acidentes, lesões autoprovocadas intencionalmente, agressões, eventos cuja intenção é indeterminada, intervenções legais e operações de guerra, complicações de assistência médica e cirúrgica, sequelas de causas externas de morbidade e mortalidade e fatores suplementares relacionados com as causas de morbidade e de mortalidade classificados em outra parte ${ }^{(1-2)}$. Atualmente, essa condição representa a terceira causa de morbidade e mortalidade no mundo, sendo responsável por 8,0\% dos óbitos registrados no ano de $2017^{(1)}$. Nesse mesmo ano, ocorreram, no Brasil, 158.657 óbitos, em decorrência de causas externas, correspondendo a $12,0 \%$ do total de óbitos. Desses, as causas mais prevalentes foram as relacionadas ao trauma por agressões $(40,2 \%)$, seguido por acidentes de transporte $(22,9 \%)$ e outras causas externas de lesões acidentais $(20,2 \%)^{(3)}$.

O trauma é considerado um abalo físico de grande impacto que resulta em uma ação abrupta ou violenta que causa danos de extensões variadas ao organismo, sendo necessário identificar o seu grau de complexidade para estabelecer a conduta terapêutica individualizada para cada paciente ${ }^{(4-6)}$. A quantidade de energia envolvida no trauma pode caracterizar o politraumatismo e resultar em lesões graves que ameacem a vida ${ }^{(4,7,8-11)}$.

$O$ acesso precoce ao atendimento adequado à pessoa vítima de trauma, ainda em ambiente préhospitalar, pode reduzir mortalidade e sequelas ${ }^{(7-}$ 9). As lesões traumáticas podem causar rápida deterioração do organismo humano e, por isso, acredita-se que a mortalidade e morbidade aumenta, drasticamente, caso a vítima não receba tratamento médico definitivo em um Centro de Trauma (CT) em até uma hora da ocorrência do trauma ${ }^{(6,9-10)}$.

Atendimentos em locais distantes de grandes centros dificultam a chegada em centros especializados no atendimento a essas vítimas ${ }^{(11)}$. Nesse sentido, o transporte aéreo surge como uma das estratégias que favorece o acesso precoce a unidades de maior complexidade e que dispõem de bloco cirúrgico, terapia intensiva e profissionais qualificados para intervir em situações específicas que agravam a saúde, melhorando expressivamente a sobrevida e qualidade de vida desses pacientes ${ }^{(9-10,12)}$.
No final da década de 1940, foram efetivados os Serviços de Helicópteros de Emergência Médica (SHEM), possibilitando aos hospitais uma nova modalidade de transporte de pacientes, quando o modo terrestre era ineficaz ou inviável $\left.\right|^{(9-10,12-13)}$. Os SHEM, quando comparados ao transporte terrestre, apresentam vantagens como o aumento na sobrevida dos pacientes, sendo impulsionado pelo tempo de transporte mais rápido, equipe de saúde mais experiente, recursos avançados e acesso precoce ao $\mathrm{CT}^{(9-10,12)}$. Apesar das vantagens, destacam-se como limitações o custo elevado, espaço pequeno da cabine, menor número de aeronaves e grande susceptibilidade a interferências climáticas ${ }^{(10,12-13)}$.

Em 2011, no Brasil, o Ministério da Saúde instituiu a Rede de Atenção às Urgências e Emergências (RUE) do Sistema Único de Saúde (SUS). A RUE reconhece o trauma como uma de suas prioridades e enfatiza a ampliação do acesso e acolhimento de casos agudos em todos os pontos de atenção, bem como a integração entre eles, com o intuito de garantir universalidade, equidade e integralidade das ações e atendimentos, por meio da linha de cuidado traumatológica ${ }^{(5-7)}$. Esta, por sua vez, tem como objetivo habilitar centros de trauma, diminuir a morbimortalidade, desenvolver ações voltadas à vigilância e prevenção, estabelecer a rede de atendimento hospitalar ao trauma, fortalecendo, dessa forma, a própria política, bem como o acesso regulado e qualificado em todos os pontos de atenção da rede $e^{(6-7)}$.

Além do investimento em CT, a RUE propôs a regionalização e ampliação do Serviço de Atendimento Móvel de Urgência (SAMU-192), com acréscimo de serviços de transporte aéreo para o atendimento pré e inter-hospitalares ${ }^{(6)}$.

Em Minas Gerais, esse tipo de serviço teve a sua ampliação, por meio do SAMU-192 e do Batalhão de Operações Aéreas do Corpo de Bombeiros Militar de Minas Gerais, o que expandiu 0 acesso a essa modalidade de transporte ${ }^{(14)}$. Nesse contexto, um dos $\mathrm{CT}$ de referência do estado tem recebido vítimas de trauma via transporte aéreo. Sabe-se que essas vítimas possuem prioridade de transporte, pois tendem a apresentar quadros mais graves, mas não se conhece os resultados dos atendimentos às mesmas, tampouco o seu desfecho. Dessa forma, questionam-se quais as características e os desfechos dos atendimentos às vítimas de trauma admitidas, via transporte aéreo, em um hospital público de grande porte de referência estadual para urgência e emergência. 
Ressalta-se que o enfermeiro compõe a equipe multiprofissional que presta assistência à vítima de trauma e tem a função de coordenar a equipe de enfermagem, além de planejar e participar da assistência a ser prestada, em um cenário, em que o tempo entre a vida e a morte é tênue.

Diante do exposto, foi desenvolvido este estudo que teve como objetivo descrever as características dos atendimentos às vítimas de trauma admitidas em um pronto- socorro de grande porte via transporte aéreo.

Espera-se que este estudo contribua como referência para futuros investimentos em serviços de emergências hospitalares, possíveis ajustes em fluxos de atendimento, além de auxiliar no fortalecimento das estratégias e políticas públicas de prevenção ao trauma, bem como para avaliação do serviço com o intuito de aumentar a resolutividade e qualidade dos atendimentos a essa clientela, aumentando, dessa maneira, as suas chances de sobrevida ${ }^{(5,7)}$.

\section{MÉTODO}

Trata-se de estudo transversal,
retrospectivo, desenvolvido no pronto

atendimento (PA) de um hospital público de grande porte, inserido no SUS, localizado em Belo Horizonte, capital do estado de Minas Gerais. O hospital é referência no atendimento a pacientes em situação de urgência, vítimas de politraumatismos, grandes queimaduras, intoxicações e situações clínicas e/ou cirúrgicas com risco de óbito, também dispõe de um plano de atendimento a incidentes com múltiplas vítimas ${ }^{(15)}$.

A população foi composta pelos prontuários informatizados de todos os pacientes que deram entrada no hospital, via transporte aéreo, no ano de 2018. Foram incluídos todos os prontuários informatizados de pacientes vítimas de trauma admitidos no hospital, via transporte aéreo e que receberam atendimento inicial na sala de reanimação do PA.

No período do estudo, foram realizadas 143 admissões de pacientes via transporte aéreo. Desses, cinco apresentavam motivo clínico e 31 não possuíam dados no sistema informatizado do hospital, apenas ficha de registro. Assim, a amostra foi composta por 107 prontuários.

As variáveis elencadas para o estudo estão apresentadas no Quadro 1.

Quadro 1 - Variáveis elencadas para o estudo. Belo Horizonte -MG -Brasil - 2018.

\begin{tabular}{|c|c|}
\hline \multirow{3}{*}{ Dados de identificação } & Variáveis do estudo \\
\hline & \multirow{2}{*}{$\begin{array}{l}\text { Sexo (feminino/masculino) } \\
\text { Idade (em anos) }\end{array}$} \\
\hline & \\
\hline Dados do acidente & Mecanismo de trauma \\
\hline \multirow{3}{*}{ Dados do transporte } & $\begin{array}{l}\text { Mecanismo de trauma } \\
\text { Responsável pelo transporte aéreo (serviço público/serviço privado) }\end{array}$ \\
\hline & Tempo de voo (em minutos) \\
\hline & Procedência do paciente \\
\hline \multirow{4}{*}{ Dados do atendimento hospitalar } & Mês da admissão \\
\hline & Dia da semana \\
\hline & $\begin{array}{l}\text { Turno de atendimento: } \\
\text { - Madrugada (00:00-11:59) } \\
\text { - } \text { Matutino (06:00-11:59) } \\
\text { - } \text { Vespertino (12:00-18:59) } \\
\text { - Noturno (19:00-23:59) }\end{array}$ \\
\hline & $\begin{array}{l}\text { Classificação de Risco segundo Sistema de Triagem de Manchester: } \\
\text { - Queixa principal } \\
\text { - } \text { Discriminadora } \\
\text { - Nível de prioridade }\end{array}$ \\
\hline \multirow{2}{*}{$\begin{array}{l}\text { Avaliação inicial à admissão segundo } \\
\text { mnemônico } A B C D E\end{array}$} & $\begin{array}{l}\text { A- Abertura das vias aéreas e controle da coluna cervical: } \\
\text { - Perviedade } \\
\text { - Dispositivo de desobstrução das vias aéreas } \\
\text { - Uso de colar cervical }\end{array}$ \\
\hline & $\begin{array}{l}\text { B- } \text { Respiração: } \\
\text { - Ventilação espontânea }\end{array}$ \\
\hline
\end{tabular}


Quadro 1 - Variáveis elencadas para o estudo. Belo Horizonte -MG -Brasil - 2018.

\begin{tabular}{|c|c|}
\hline & Variáveis do estudo \\
\hline \multirow{4}{*}{$\begin{array}{l}\text { Avaliação inicial à admissão segundo } \\
\text { mnemônico ABCDE }\end{array}$} & $\begin{array}{l}\text { - Administração de oxigênio } \\
\text { - Dispositivo de administração de oxigênio } \\
\text { - Frequência respiratória } \\
\text { - } \text { Ausculta pulmonar }\end{array}$ \\
\hline & $\begin{array}{l}\text { C- } \text { Circulação: } \\
\text { - Frequência cardíaca } \\
\text { - } \text { Ausculta cardíaca } \\
\text { - Pressão arterial sistólica e diastólica } \\
\text { - Tempo de enchimento capilar }\end{array}$ \\
\hline & $\begin{array}{l}\text { D- Disfunção neurológica: } \\
\text { - Escala de Coma de Glasgow na cena e admissão } \\
\text { - Características pupilares na cena e admissão } \\
\text { - Reflexo fotomotor direto na cena e admissão } \\
\text { - Relato de perda da consciência }\end{array}$ \\
\hline & $\begin{array}{l}\text { E- Exposição e ambiente: } \\
\text { - Imobilização em prancha longa } \\
\text { - Tipos de lesões e regiões corpóreas atingidas }\end{array}$ \\
\hline \multirow{6}{*}{ Propedêutica e terapêutica } & $\begin{array}{l}\text { Dispositivos de assistência: } \\
\text { - } \quad \text { Punção de acesso venoso periférico } \\
\text { - } \text { Monitorização de pressão intra-arterial } \\
\text { - } \quad \text { Puncterismo vesical de demora } \\
\text { - }\end{array}$ \\
\hline & Exames de imagem solicitados das primeiras $24 \mathrm{~h}$ \\
\hline & Resultados dos exames de imagem solicitados das primeiras $24 \mathrm{~h}$ \\
\hline & Reserva de hemoderivados nas primeiras $24 \mathrm{~h}$ \\
\hline & Tipo de hemoderivado transfundido nas primeiras $24 \mathrm{~h}$ \\
\hline & Terapêutica instituída (cirúrgica/conservadora) \\
\hline \multirow{4}{*}{ Desfecho } & $\begin{array}{l}\text { Desfecho no PA (alta para domicílio, Bloco Cirúrgico, Unidade de Terapia Intensiva, } \\
\text { unidade de internação, transferência para outra unidade hospitalar, óbito) }\end{array}$ \\
\hline & Tempo de permanência no PA (em horas) \\
\hline & $\begin{array}{l}\text { Desfecho hospitalar (alta para domicílio, transferência para outra unidade hospitalar, } \\
\text { óbito) }\end{array}$ \\
\hline & Tempo de permanência hospitalar (em dias) \\
\hline
\end{tabular}

Em relação ao mecanismo de trauma, os pacientes vítimas de queimaduras receberam classificação de gravidade, conforme classificação da superfície corpórea queimada (SCQ), sendo considerado: pequeno queimado (1으 e 2 o grau até $10 \%$ da SCQ), médio queimado (1o e 2 o grau entre 10 a 25\% da SCQ; 3으 grau até 10\% da SCQ, queimaduras de mãos pés e face) e grande queimado (1ㅇ e 2o grau acima de $26 \%$ da SCQ; 3 o grau acima de $10 \%$ da SCQ e/ou queimaduras de períneo, elétricas, vias aéreas e presença de comorbidades) ${ }^{(16)}$.

Os dados relacionados à classificação de risco (CR) consideraram as variáveis do Sistema de Triagem de Manchester (STM). No STM, a queixa principal aponta para a escolha de um fluxograma que indica a avaliação de sinais e sintomas, por meio de discriminadores que determinam um nível de prioridade clínica para o primeiro atendimento médico. Os níveis de prioridade do STM incluem: 1emergência (vermelho), 2-muito urgente (laranja), 3-urgente (amarelo), 4-pouco urgente (verde) e 5não urgente (azul). Para cada prioridade existe um tempo máximo de espera para o primeiro atendimento, sendo, respectivamente, 0, 10, 60, 120 e 240 minutos $^{(17)}$.

A sequência da avaliação primária dos pacientes traumatizados, deve-se basear na sequência do mnemônico ABCDE. Essas letras indicam a sequência da avaliação e direcionam o atendimento do profissional, a fim de priorizar o tratamento de lesões que ameaçam a vida dos pacientes $^{(7,18)}$.

No que se refere à avaliação neurológica, recomenda-se a Escala de Coma de Glasgow (ECG) para a avaliação do nível de consciência da vítima. Essa escala, define um escore que pode variar de três (nível de consciência muito comprometido) a 15 pontos (nível de consciência preservado). São avaliados três parâmetros (abertura ocular, melhor resposta verbal e melhor resposta motora) e a 
pontuação em cada um deles refere-se a melhor resposta apresentada pelo paciente ${ }^{(11,19)}$.

Para a coleta de dados foi elaborado um instrumento que contemplava as variáveis do estudo. A coleta foi realizada pelos pesquisadores, durante os meses de julho a dezembro de 2019 e ocorreu em duas etapas. A primeira consistiu em uma consulta a ficha de registro específica do hospital, preenchida para os pacientes que foram admitidos, via transporte aéreo, na sala de reanimação, em que foram coletados dados referentes ao registro, às características demográficas e clínicas dos pacientes, além dos dados do transporte. A partir dos dados da ficha de registro, sucedeu a segunda etapa em que foi realizada consulta aos prontuários eletrônicos disponibilizados pelo sistema de gestão informatizado do hospital para a coleta das variáveis referentes aos dados da classificação de risco, atendimento hospitalar, avaliação inicial à admissão, propedêutica, terapêutica e desfecho dos atendimentos.

Após o registro das variáveis no instrumento de coleta, as informações foram transcritas para um banco de dados criado no software Epidata 3.0, utilizando a técnica de dupla digitação para reduzir as chances de digitação errônea. Posteriormente, os dados foram exportados para o software Statistical Package for the Social Sciences (SPSS) versão 25.0 e foram submetidos à análise estatística descritiva. Para as variáveis quantitativas, foram determinadas medidas de tendência central (média e mediana) e medidas de dispersão (mínimo, máximo e intervalo interquartílico - IQ). Utilizou-se o teste de normalidade Kolmogorov-Smirnov para as variáveis quantitativas para definição do uso de média ou mediana na apresentação dos dados. As variáveis categóricas foram apresentadas como frequências absolutas e relativas.

Este estudo fundamentou-se na Resolução 466/2012 e 510/16 do Conselho Nacional de Saúde. O projeto de pesquisa foi autorizado pelo Comitê de Ética da Fundação Hospitalar do Estado de Minas Gerais (FHEMIG), CAAE: 15886919.6.0000.5119, aprovado com o parecer de número: 104/2019.

\section{RESULTADOS}

Dos 107 pacientes analisados, a maioria era do sexo masculino (68-63,6\%). A idade variou de zero a 87 anos, com mediana de 32 anos (IQ:23 51).
A colisão automobilística foi o mecanismo de trauma mais frequente $(30-28,0 \%)$, seguido por capotamento veicular (23 - 21,5\%), queimaduras (17 - 15,9\%), queda de modais de transporte (13 $12,1 \%)$. Os demais apresentaram percentuais inferiores a 10,0\% e incluíram: atropelamento, agressão física e queda de altura. O transporte aéreo público pré-hospitalar foi responsável por 92 $(86,0 \%)$ transportes ao serviço. O tempo de voo variou de três a 100 minutos com mediana de 14 minutos (IQ:10-26).

Quanto à procedência, a maioria das vítimas (87 - 81,3\%) eram provenientes da cidade de Belo Horizonte e região metropolitana. A distribuição da procedência, segundo região do estado está apresentada na Figura 1.

Figura 1 - Distribuição dos pacientes vítimas de trauma admitidos via transporte aéreo segundo a procedência. Belo Horizonte - MG - Brasil, 2019. MINAS GERAIS - MESORREGIÖES

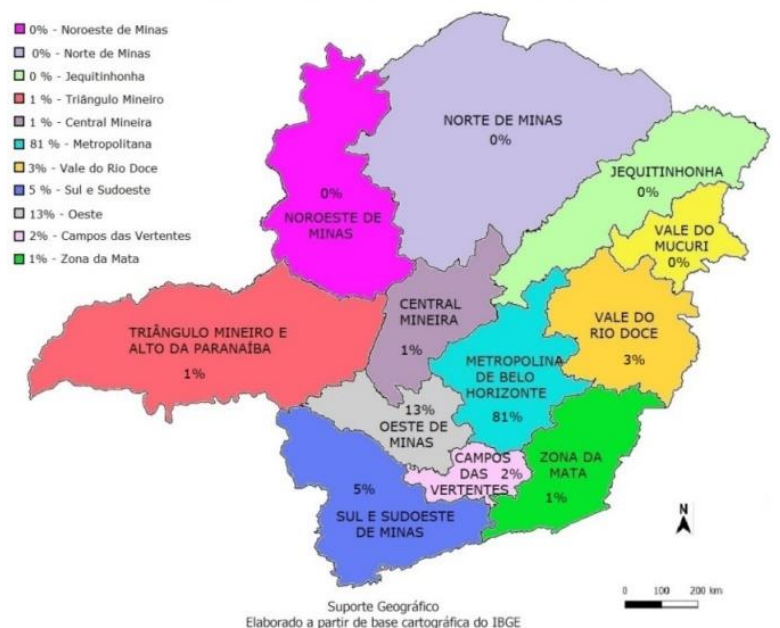

Fonte: Elaborado para fins deste estudo a partir da base cartográfica do Instituto Brasileiro de Geografia e Estatística IBGE.

\section{Dados do atendimento hospitalar}

O mês com o maior número de admissões foi outubro (20 - 18,7\%), seguido de dezembro (15,0 $14,0 \%)$ e janeiro (12 - 11,2\%). Os demais meses apresentaram percentual semelhante, todos menores do que $10,0 \%$.

No que se refere ao dia da semana, o maior número de admissões ocorreu aos sábados (23 $21,0 \%$ ) e domingos $(23-21,0 \%)$, seguidos por segunda-feira $(16-15,0 \%)$ e terça-feira (15 $14,0 \%)$. Os demais dias de semana apresentaram percentual menor do que $10 \%$. O turno de atendimento com maior número de admissões foi o vespertino (75 - 70,1\%), seguido por matutino (19-17,8\%) e noturno 13 (13-12,1\%). 
Não houve registros de dados da CR em 46 $(43,0 \%)$ prontuários. Em relação a queixa principal, 15 (24,6\%) foram decorrentes de colisão automobilística, $12(19,7 \%)$ queimaduras, 11 $(18,0 \%)$ capotamento veicular, $11(18,0 \%)$ colisão motociclística e os demais apresentaram percentual menor do que 10,0\% para a variável. Ressalta-se que houve relato de mais de uma queixa principal por um mesmo paciente.

O fluxograma 'Trauma maior' foi descrito em 19 (31,1\%) prontuários, seguido por 'Queimaduras' $(6-9,9 \%)$. Dentre os pacientes classificados, 36 $(59,0 \%)$ não tiveram registro de fluxograma. Quanto ao discriminador, 'mecanismo de trauma significativo' foi o mais frequente $(11-18,0 \%)$, seguido de 'respiração inadequada' ( $7-11,5 \%)$, os demais (9 - 14,8\%) apresentaram percentuais menores do que $10 \%$. Dentre os pacientes classificados, 34 (55,7\%) não tinham discriminador registrado. O nível de prioridade clínica variou entre vermelho-emergência (34 - 55,7\%) e laranja (25 - 41,0\%) e em dois prontuários (3,3\%) não havia o registro do nível de prioridade clínica.

\section{Avaliação inicial à admissão}

A- Via aérea e controle de coluna cervical

A via aérea (VA) estava pérvia em 106 $(99,1 \%)$ casos, porém houve um $(0,9 \%)$ relato de obstrução de VA que estava relacionada à intubação esofágica. No que tange ao dispositivo de desobstrução de VA, a utilização do tubo orotraqueal foi descrita em 59 (55,0\%) atendimentos.

A utilização do colar cervical como dispositivo de alinhamento e estabilização da coluna cervical foi descrito em $83(77,6 \%)$ prontuários e 14 pacientes $(13,1 \%)$ não fazia seu uso à admissão na sala de emergência. Em dez prontuários (9,3\%) não havia relato da utilização desse dispositivo.

\section{B- Ventilação}

A ventilação espontânea estava comprometida em 59 (55,0\%) pacientes e 73 $(68,2 \%)$ receberam oxigênio suplementar. Dentre os dispositivos de administração de oxigênio, predominou o ventilador mecânico (58 -79,5\%), seguido por máscara facial com reservatório não reinalante (10 - 13,7\%) e cateter nasal ( 5 - 6,8\%).

A frequência respiratória (FR) variou de 0 a 32 incursões respiratórias, por minuto (irpm), com mediana de 18irpm e (IQ: 16 - 20). Mais da metade dos pacientes $(67-62,6 \%)$ apresentou murmúrios vesiculares fisiológicos (MVF) à ausculta pulmonar,
$12(11,2 \%)$ apresentaram MVF diminuídos, dois $(1,9 \%)$, crepitações difusas $1(0,9 \%)$ roncos difusos bilaterais e em 25 (23,4\%) prontuários não havia registro dessa variável.

\section{C- Circulação}

A frequência cardíaca $(F C)$ variou de zero a 173 batimentos por minuto (bpm) e apresentou mediana de 96bpm e (IQ: 79 - 110). Na ausculta cardíaca as bulhas normorritmicas e normofonéticas em dois tempos foram descritas $68(64,0 \%)$ vezes e em 39 prontuários $(36,0 \%)$ não havia descrição dessa variável.

A pressão arterial sistólica (PAS) variou de 0 a $180 \mathrm{mmHg}$, com mediana de $120 \mathrm{mmhg}$ (IQ: 103,0-130,0). A pressão arterial diastólica (PAD) variou de 0 a $120 \mathrm{mmHg}$, com mediana de $71 \mathrm{mmHg}$ (IQ: 58 - 81).

O tempo de enchimento capilar estava menor do que três segundos em $45(42,0 \%)$ casos e maior do que três segundos em cinco (4,7\%). Em mais da metade dos prontuários (57 - 53,3\%) não havia registro dessa variável.

\section{D- Disfunção neurológica}

A ECG na cena variou entre 3 e 15 pontos, com mediana de 10 pontos (IQ: 6 -15). Ressalta-se que não houve registro desse dado em $48(44,0 \%)$ prontuários.

As características das pupilas na cena foram descritas como isocóricas em 23 (21,5\%) pacientes, anisocóricas em três $(2,8 \%)$ e midriáticas em um $(0,9 \%)$. Em 80 prontuários $(74,7 \%)$ não havia descrição dessa variável. No que se refere ao teste do reflexo fotomotor direto, na cena, 23 pacientes $(21,5 \%)$ apresentavam reatividade pupilar bilateral e em 84 prontuários $(78,5 \%)$ não havia registro dessa informação.

O relato de perda de consciência na cena foi descrito a $24(22,4 \%)$ pacientes, $16(15,0 \%)$ não perderam a consciência e não foi informado em 67 (62,6\%) prontuários.

A ECG na admissão variou entre 3 a 15, com mediana de 15 (IQ: 9-15), porém não houve registro em 43 (40,2\%) prontuários.

As características das pupilas na admissão foram: isocóricas em 60 (56,1\%) pacientes, mióticas em 16 (14,9\%), anisocóricas em 9 (8,4\%) e midriáticas em 8 (7,5\%). Ressalta-se ausência de registro dessa variável em $14(13,1 \%)$ prontuários. No que se refere ao reflexo fotomotor direto, 71 $(66,3 \%)$ pacientes apresentavam reatividade pupilar bilateral, oito (7,5\%) ausência de 
reatividade pupilar, bilateralmente, em três $(2,8 \%)$ a reatividade estava ausente unilateralmente e em $25(23,4 \%)$ prontuários não havia registro dessa variável.

\section{E- Exposição e ambiente}

A maioria $(91-85,0 \%)$ dos pacientes foram admitidos imobilizados em prancha longa, quatro $(4,0 \%)$ receberam imobilização em membros superiores e seis $(6,0 \%)$ imobilização em membros inferiores.

Os dados referentes aos tipos de lesões e regiões corpóreas atingidas estão apresentados na Tabela 1.

Tabela 1 - Tipos de lesões e regiões corpóreas atingidas de pacientes vítimas de trauma admitidas em um pronto socorro via transporte aéreo. Belo Horizonte - MG - Brasil 2018. ( $N=107)$.

\begin{tabular}{lcc}
\hline Tipos de lesões & N & $\%$ \\
\hline Escoriações & 36 & 33,6 \\
Ferida corto contusa & 33 & 30,8 \\
Queimadura de 20 e 30 graus & 19 & 17,7 \\
Tatuagem traumática & 12 & 11,2 \\
Blefarohematoma & 11 & 10,2 \\
Sem registro no prontuário & 10 & 9,3 \\
Regiões corpóreas atingidas & & \\
Membros & 71 & 66,3 \\
Crânio & 62 & 57,9 \\
Tórax & 44 & 41,1 \\
Face & 18 & 16,8 \\
Abdome & 18 & 16,8 \\
Coluna cervical & 12 & 11,2 \\
Coluna vertebral & 11 & 10,2 \\
Sem registro no prontuário & 4 & 3,7 \\
\hline
\end{tabular}

Fonte: elaborada pelos autores.

Dentre os pacientes queimados, observouse que a maioria $(16-84,2 \%)$ foi classificada como grande queimado.

\section{Propedêutica e terapêutica}

Quanto aos procedimentos realizados na admissão dos pacientes, o mais frequente foi a punção de acesso venoso periférico (AVP), sendo realizada em 93 pacientes $(87,0 \%)$, seguido de cateterismo vesical de demora em 61 (57,0\%), punção de cateter venoso central (CVC) em 32 $(30,0 \%)$ e monitorização de pressão intra-arterial em 25 pacientes $(23,0 \%)$.

Os dados referentes aos exames de imagem solicitados nas primeiras $24 \mathrm{~h}$ e os seus respectivos resultados estão apresentados na Tabela 2 .
Tabela 2 - Distribuição dos exames de imagem e respectivos resultados solicitados nas primeiras $24 \mathrm{~h}$ para os pacientes vítimas de trauma admitidas em um pronto socorro via transporte aéreo. Belo Horizonte - MG - Brasil - 2018. ( $N=107)$.

\begin{tabular}{lcc}
\hline Exames de imagem solicitados primeiras $24 \mathrm{~h}$ & $\mathrm{~N}$ & $\%$ \\
\hline Tomografia & 78 & 72,9 \\
Radiografia & 72 & 67,3 \\
FAST abdominal & 46 & 43,0 \\
Tomografia & & \\
Crânio & 68 & 63,5 \\
Coluna cervical & 66 & 61,7 \\
Tórax & 49 & 45,8 \\
Abdome & 48 & 44,9 \\
Pelve/Bacia & 48 & 44,9 \\
Coluna lombo sacra & 30 & 28,0 \\
Coluna torácica & 28 & 26,2 \\
Face e seios nasais & 11 & 10,3 \\
Radiografia & & \\
Tórax & 60 & 56,0 \\
Pelve/bacia & 32 & 30.0 \\
Braço & 23 & 21,5 \\
Perna & 20 & 18,7 \\
Coxa & 16 & 14,9 \\
Joelho & 11 & 10,3 \\
Resultados dos exames de imagem & & \\
Hemorragia intracraniana & 10 & 9,3 \\
Fratura de membros & 3 & 2,8 \\
Fratura de coluna & 30 & 28,0 \\
Fratura de arcos costais & 29 & 27,1 \\
Pneumotórax/Hemotórax & 18 & 16,8 \\
Fratura do crânio & 14 & 15,9 \\
Fratura dos ossos da face & 12,1 \\
Contusão cerebral & 11,2 \\
Contusão pulmonar & & 10,2 \\
Sem alterações & & \\
\hline & & \\
& &
\end{tabular}

Fonte: elaborada pelos autores.

Foi solicitada reserva de hemoderivados ao banco de sangue para $59(55,1 \%)$ vítimas, sendo: concentrado de hemácias ( 59 - 55,1\%), plasma (53 - 49,5\%), plaquetas (23 - 21,5\%) e crioprecipitado (8 - 6,5\%). Foram efetivamente transfundidos: concentrado de hemácias ( $25-23,4 \%$ ), plasma (10 - 9,3\%), plaquetas (5 - 4,7\%) e crioprecipitado (8 $6,5 \%)$.

O registro do tipo de terapêutica instituída estava ausente em oito prontuários (7,5\%). Predominou a abordagem cirúrgica $(65-60,7 \%)$ seguida pela conservadora 34 (31,8\%). O tempo de permanência dos pacientes no PA variou de 16 minutos a 269 horas a 25 minutos com mediana 9 
horas e 52 minutos, (IQ: 3 horas e 34 minutos -29 horas e 55 minutos).

Como desfecho do PA, metade ( $54-50,5 \%)$ foi encaminhada ao bloco cirúrgico, 13 (12,1\%) evoluíram a óbito, $12(11,02 \%)$ receberam alta para o domicílio, 11 (10,3\%) foram encaminhados para a UTI, nove $(8,4 \%)$ para uma unidade de internação e oito $(7,5 \%)$ foram transferidos para outra unidade hospitalar.

O tempo de internação hospitalar variou de 0 a 301 dias com mediana 7 dias (IQ: 1,5-33,0). Como desfecho hospitalar, mais da metade $(61-$ $57,0 \%)$ recebeu alta para o domicílio, 28 (26,2\%) evoluíram a óbito e $18(16,8 \%)$ foram transferidos para outra unidade hospitalar.

\section{DISCUSSÃO}

A predominância de homens e mecanismo de trauma automobilístico vem sido destacada em outros estudos com usuários vítimas de trauma, além disso é um padrão que se repete em âmbito nacional $\left.\right|^{(4-8,10,13)}$. Esse achado pode estar relacionado com o aumento do consumo de álcool e drogas, a maior facilidade na aquisição de veículos, bem como a fatores relacionados à cultura e imprudência em relação às leis de trânsito ${ }^{(4-5,7,8,13)}$.

A mediana de idade dos pacientes foi de 32 anos, idade que representa uma população economicamente ativa ${ }^{(4,6-8)}$. Características dessa faixa etária, como inexperiência ao volante, imaturidade, associação entre o uso de drogas e direção, além de outros comportamentos de risco, são alguns dos fatores que podem ter ligações com as estatísticas deste e de outros estudos que apontam o mesmo resultado para variável ${ }^{(5-}$ $6,8,10,13,20)$. O paciente vitimado também pode apresentar interrupção de suas atividades produtivas, perda econômica, deixando de gerar renda, sendo necessária a utilização do serviço previdenciário ${ }^{(5,8,20)}$.

Metade dos transportes aéreos foram realizados em 14 minutos ou menos. O atendimento, em um curto período de tempo após a ocorrência de lesões traumáticas, pode ser o maior fator associado à redução da mortalidade, pois quanto mais rápido se resgatam as vítimas, tão logo o diagnóstico é estabelecido e o tratamento é iniciado $^{(4,9,10)}$. Dessa maneira, o transporte aéreo pode contribuir na redução do tempo de resposta e acesso ao serviço médico especializado ${ }^{(4,9-10,12,13)}$.

Estudo que validou um protocolo de triagem médica ao paciente vítima de trauma para definição da necessidade de remoção aérea, evidenciou aumento da taxa de sobrevivência em $22 \%$ daqueles cuja modalidade de transporte foi indicada ${ }^{(9)}$. Embora o transporte aéreo tenha demostrado benefícios no que se refere à taxa de sobrevivência, a seleção dos pacientes eleitos a essa mobilidade de transporte ainda é um desafio(9-10)

Faz-se necessária a transferência dos pacientes em estado grave em um tempo hábil, uma vez que em o tratamento definitivo para os casos de choque hipovolêmico hemorrágico demanda intervenção cirúrgica e/ou da infusão de hemocomponentes, procedimentos atualmente disponíveis somente no nível terciário(6,9-10,21). Sabe-se que a infusão de coloides e cristaloides no atendimento pré-hospitalar é intervenção temporária na tentativa de garantir a perfusão adequada dos órgãos, entretanto, não garante transporte de oxigênio adequado nos casos de perdas sanguíneas (21-22).

Quanto ao predomínio da região metropolitana de Belo Horizonte na análise da procedência dos pacientes, deve-se levar em consideração a regionalização dos serviços de saúde, no âmbito do SUS, com a disponibilização de centros de atendimento com vários níveis de atenção em pontos estratégicos ${ }^{(20)}$. Belo Horizonte é considerada polo estadual de Minas Gerais (MG), sendo também polo macrorregional de referenciação para as regiões administrativas metropolitana de $\mathrm{MG}^{(23)}$.

Os meses com maior prevalência de admissões, foram os relacionados a feriados prolongados e férias escolares, períodos em que aumentam, expressivamente, o número de viagens e, assim, maior chance de acidentes automobilísticos. A ocorrência de maior número de admissões nos finais de semana e no período vespertino foi dado semelhante em outros estudos sobre o tema ${ }^{(4,8,13,20)}$. Infere-se que a prevalência das admissões, nesses dias, possa estar relacionada ao consumo de bebida alcóolica, que está associado a alterações cognitivas, comportamentos violentos e condutas de trânsito inadequadas ${ }^{(5,8,13,20)}$.

A baixa incidência de admissões no período noturno pode estar relacionada ao fato de, grande parte das aeronaves necessitarem voar em situações climáticas favoráveis e não trafegarem por instrumento, o que restringe o tráfego, nesse horário e durante a ocorrência de chuvas ${ }^{(12,22)}$. 
Quanto aos dados da classificação de risco, o fato de quase metade não possuir esses registros pode ser atribuído à forma de entrada do paciente na instituição, ou seja, não passa pelo espaço físico destinado à classificação o que pode implicar na ausência desse importante registro.

A maior frequência de acesso aos fluxogramas 'trauma maior' e' queimadura' vai de encontro com os mecanismos de trauma mais prevalentes e queixas principais apresentadas pela população estudada. A maior frequência dos discriminadores 'mecanismo de trauma significativo' e 'respiração inadequada' foram condizentes com os níveis de prioridade clínica que indicavam atendimento médico imediato ou em até dez minutos. Em decorrência da insuficiência de registros da $C R$, não se pode afirmar a ocorrência de um maior nível de gravidade, nesses pacientes, mas com base nos pacientes classificados, foi constatada a necessidade de atendimento médico imediato, especializado e recursos de tratamento mais avançados em um centro de referência, o que corrobora com a indicação do transporte aeromédico.

Em relação à avaliação inicial da vítima de trauma, mais da metade foi admitida com prótese de via aérea. $O$ uso de via aérea artificial é indicado quando as técnicas manuais de desobstrução da via aérea falham, em pacientes inconscientes e/ou com depressão acentuada do nível de consciência, podendo ser considerado quando atinge escore $\leq 8$ na $\operatorname{ECG}^{(7,22,24)}$. Esse fato aponta para o alto grau de complexidade desses pacientes. A intubação orotraqueal, por sua vez, é o método preferido para controle de vias aéreas, pois diminui, significativamente, o risco de broncoaspiração, facilita a aspiração profunda, previne insuflação gástrica, além de permitir uma via de acesso para administração de medicamentos ${ }^{(21)}$.

O uso do colar cervical associado a blocos laterais para imobilização da cabeça, objetiva a prevenção de lesão secundária à medula cervical ${ }^{(7)}$. A ausência desses dispositivos em uma parcela de pacientes pode estar relacionada às discussões evidências científicas recentes que defendem o uso discriminado desses dispositivos nos casos realmente indicados ${ }^{(18)}$. A não indicação do colar cervical se dá em pacientes não intoxicados, sem dores cervical e/ou rigidez cervical, sem déficit neurológico e sem fatores distratores, como dor intensa em outras regiões do corpo ${ }^{(07,18)}$. Também pode estar relacionado à avaliação pregressa em outras instituições de saúde que descartassem lesões cervicais, porém este estudo não traz informações suficientes para confirmar essa hipótese.

A administração de oxigênio suplementar com fluxo de 10L/min na vítima de trauma é indicada com o objetivo de manter oxigenação tecidual maior que $95 \%$, sendo um dos objetivos do atendimento inicial a vítima de trauma ${ }^{(7,22,24)}$. A administração de oxigênio sob máscara facial ou por meio do ventilador mecânico é indicada a todos os pacientes traumatizados com ou sem respiração espontânea que serão submetidos a transporte aéreo, em decorrência da possibilidade de hipóxia, durante o voo, causado pela diminuição da pressão parcial de $\mathrm{O} 2$ com elevação da altitude, o que corrobora com dados deste estudo ${ }^{(22)}$.

Metade dos pacientes apresentavam-se taquipneicos o que pode estar relacionado também aos parâmetros do ventilador, pois $79,5 \%$ dos pacientes estavam em ventilação mecânica. A taquipneia é um dado vital que indica insuficiência de oxigenação tecidual e/ou aumento do metabolismo tecidual o que repercute em aumento da respiração celular anaeróbia e, consequentemente, aumento do $\mathrm{CO}_{2}(7,21,24)$. $\mathrm{O}$ centro respiratório reconhece o nível elevado de $\mathrm{CO} 2$ e aumenta a FR, na tentativa de eliminar esse gás em excesso ${ }^{(7,21,24)}$. Logo, a FR é um dado vital que pode indicar, precocemente, déficits na perfusão, na oxigenação tecidual ou em $\operatorname{ambos}^{(21,24)}$.

Nos casos em que houve diminuição da ausculta de murmúrios vesiculares fisiológicos, pode-se inferir que a dor decorrente de trauma de tórax pode acarretar na diminuição da expansibilidade torácica e, consequentemente, na diminuição desses ruídos à ausculta ${ }^{(12,22)}$. Além disso, a falta de informações referente a essa variável pode dificultar a detecção precoce de pneumotórax e/ou hemotórax e o estabelecimento de diagnóstico e intervenções imediatas $^{(4,12-13,22)}$.

O pneumotórax hipertensivo, pneumotórax aberto, hemotórax maciço e tórax instável necessitam de diagnóstico precoce e intervenções imediatas, pois podem comprometer a ventilação e gerar risco imediato de morte ${ }^{(4,12,22)}$.

Quanto ao estado circulatório, percebeu-se que mais da metade dos pacientes apresentavam sinais de estabilidade hemodinâmica. Os valores elevados das pressões sistólica e diastólica em $50 \%$ dos casos podem ser relacionado à exposição e à manipulação das vítimas e à situação de estresse, 
por receberem cuidados em um ambiente desconhecido. Entretanto, salienta-se que pacientes vítimas de politraumatismos estão mais propensos à ocorrência de choque circulatório, em especial o hipovolêmico(21).

Em pacientes vítimas de trauma, a avaliação do nível de consciência pela escala de coma de Glasgow (ECG), a avaliação pupilar, o reflexo fotomotor e a história de perda de consciência determinam o nível de função cerebral e predizem nível de gravidade e sobrevida ${ }^{(7)}$. Assim, é essencial conhecer qual o resultado de sua avaliação na cena e na admissão, pois esses dados repercutem, diretamente, na prioridade de transporte desses pacientes, no acionamento de recursos como o transporte aéreo, bem como em manejo intrahospitalar ${ }^{(6,12)}$.

Neste estudo, metade dos pacientes apresentaram escore da ECG menor do que 10 o que indica quadros graves com potencial necessidade de prótese de via aérea. Sabe-se que a intubação orotraqueal é indicada para pacientes com rebaixamento do nível de consciência (ECG $\leq 8)$ e que não protegem via aérea(7). Portanto, esse fato pode justificar a prevalência de admissões de pacientes com intubação orotraqueal e ventilação mecânica(12,22,24).

A falta de registro do escore da ECG nas admissões pode ser explicada pela possibilidade dos pacientes terem recebido algum tipo de sedação no atendimento pré-hospitalar, para a realização de intubação orotraqueal e ajuste da ventilação. Essa conduta inviabiliza a utilização da ECG, sendo indicada, para esses casos, utilização de escalas que avaliem nível de sedação, não descritas na maioria das admissões. Diante dessas circunstâncias, ressalta-se a relevância de registro do escore da ECG na avaliação inicial do atendimento pré-hospitalar. Esse dado pode auxiliar no manejo adequado desse cliente em nível intra-hospitalar ${ }^{(6,7,12,22)}$.

A avaliação das pupilas, na admissão dos pacientes, auxilia para a definição do nível de gravidade, conduta neurocirúrgica, bem como no prognóstico das vítimas de $\operatorname{TCE}^{(7,22)}$. O rebaixamento do nível de consciência associado à anormalidade no reflexo pupilar pode indicar lesões encefálicas potencialmente fatais ${ }^{(7)}$. Neste estudo, bem menos da metade dos prontuários tinham registro dessa avaliação. Diante da gravidade e necessidade de abordagem precoce é um resultado que precisa ser divulgado para que $s$ serviços envolvidos repensem os protocolos de atendimento e capacitação das equipes de saúde.

Quanto a imobilização dos pacientes, apesar das recomendações de uso de prancha longa e imobilização cervical a todos os pacientes vítimas de trauma ${ }^{(11)}$, é possível que alguns pacientes tenham recebido primeiro atendimento em outras instituições, sendo realizados exames de imagem e avaliação médica que excluíram lesões de coluna, o que justificaria o não uso desses dispositivos para o transporte aéreo.

Estudo que avaliou intervenções em vítimas de trauma, no período que antecede ao transporte aeromédico ${ }^{(22)}$, apresentou dados de imobilização semelhantes a este estudo. Sabe-se que manter o paciente posicionado em prancha longa, durante o transporte aéreo, promove estabilização da vítima, diminui o risco de lesões medulares secundárias, diminui o desconforto, aumenta a segurança durante o voo e minimiza os efeitos gravitacionais $^{(18,22)}$.

Os membros inferiores e superiores, o crânio e o tórax, foram as regiões corpóreas mais atingidas. Os membros inferiores comportam os ossos mais longos do corpo, portanto, após uma colisão veicular, são os ossos em que recebem maior dissipação de energia ${ }^{(7,11)}$. Por outro lado, os membros superiores estão relacionados aos mecanismos de defesa do ser humano como tentativa de amenizar o impacto ${ }^{(11)}$. Estudo de revisão sobre a temática, desta pesquisa, mostrou que as lesões mais frequentes foram as fraturas e traumatismo cranioencefálico $(\mathrm{TCE})^{(8)}$. O TCE também é apresentado como principal mecanismo de trauma em estudo documental, qualitativo, retrospectivo que caracteriza o perfil das ocorrências em um transporte aeromédico ${ }^{(13)}$.

As queimaduras foram o terceiro tipo de lesão mais frequente na amostra estudada sendo, a maioria, grandes queimados. Sabe-se que a instituição pesquisada é referência estadual no atendimento a essa clientela, dispondo de uma unidade de tratamento de queimados com unidade de cuidados intensivos e bloco cirúrgico específico para esses pacientes. Essa característica permite cuidado em ambiente diferenciado e maior proteção a infecções secundárias, complicação essa de grande risco, em decorrência do comprometimento da pele em corporais áreas extensas ${ }^{(16)}$.

Quanto aos procedimentos realizados, o acesso venoso periférico (AVP) foi o mais prevalente. Trata-se de uma via de administração 
rápida de fluídos e medicamentos ${ }^{(22)}$. A necessidade da sua utilização em vítimas de trauma é justificada pela chance de choque e da necessidade da infusão de medicamentos endovenosos e hemoderivados ${ }^{(7)}$.

Os exames de imagem foram realizados em mais da metade dos pacientes, sendo a tomografia e a radiografia os mais prevalentes. Sabe-se que os resultados dos exames de imagem são definidores de diagnóstico, indicam nível de gravidade das lesões e também auxiliam no estabelecimento de condutas e tratamento imediato ${ }^{(19)}$. Os custos da realização de tomografia são altos, além disso, esse tipo de exame não está disponível em todos os níveis de atenção, tampouco em todas as instituições hospitalares, justificando, mais uma vez, a necessidade de transporte precoce desses pacientes para uma instituição de referência ${ }^{(8,19)}$.

A hemorragia intracraniana, a fratura de membros e a fratura de coluna foram os resultados de exames de imagem mais frequentes. Esses resultados estão de acordo com a indicação do transporte aéreo, bem como no nível de gravidade dessas vítimas e na necessidade de cuidado especializado(11).

O tipo de terapêutica mais frequente foi a cirúrgica, dado este que comprova a necessidade de acesso a unidades de alta complexidade ${ }^{(11,19)}$.

Quanto ao tempo de internação, metade dos pacientes permaneceram na instituição por período inferior a sete dias. Estudo semelhante realizado em um CT, do Rio de Janeiro, apresentou tempo médio de internação superior $(11,4 \text { dias })^{(6)}$. Sabe-se que períodos de internação estendidos predispõem à ocorrência de eventos adversos e outras complicações. Esses fatores, associados à constante sobrecarga do serviço podem onerar o sistema de saúde e impactar no processo produtivo, econômico e social do país ${ }^{(6,8)}$.

Estudo realizado nos EUA que comparou o tempo médio de permanência de pacientes transportados, pelo transporte aéreo e por via terrestre, evidenciou que os pacientes com acesso ao transporte aéreo tiveram maior média de internação hospitalar e em Terapia Intensiva dado que demonstra a gravidade e justificativa de acesso precoce a serviços especializados.

O percentual de óbitos como desfecho da internação hospitalar encontrado, neste estudo, é um dado preocupante. Estudo semelhante aponta taxa de óbito menor ${ }^{(6)}$. Entretanto, destaca-se que o acesso ao CT via transporte aéreo pode ter contribuído para a alta hospitalar que ocorreu em mais da metade das vítimas. Estudo multicêntrico retrospectivo que avaliou a diferença do desfecho entre pacientes vítimas de trauma, transportados por via terrestre e aérea realizado nos Estados Unidos da América (EUA), evidenciou que os pacientes que foram transportados por helicóptero eram $57 \%$ menos propensos a morrer do que os transportados por ambulâncias terrestres (IC95\%, p<0,001) ${ }^{(10)}$.

Ressalta-se que este estudo não avaliou a qualidade de vida, tampouco as capacidades funcionais após a alta. Sugere-se a realização de estudos com delineamentos mais robustos que avaliem a sobrevida dessa população, após a alta hospitalar, bem como qualidade de vida e incapacidades funcionais adquiridas em decorrência do trauma(5).

Embora os resultados evidenciados pelo estudo sejam relevantes, o mesmo apresentou limitações relacionadas ao registro incompleto e incorreto de dados essenciais para levantamento estatístico, tanto no formulário dos pacientes admitidos no heliponto quanto no prontuário informatizado da instituição. A insuficiência de registro dos profissionais envolvidos no atendimento a essas pacientes reflete que a assistência nem sempre é devidamente documentada, o que compromete diversos processos institucionais, como a reformulação de protocolos e fluxos e de estudos de abordagem retrospectiva.

Em razão da ausência significativa de registros referentes aos hábitos de vida, principalmente quanto ao uso de drogas lícitas e ilícitas, não foi possível fazer associações entre os acidentes de trânsito e sua utilização a essa população específica.

\section{CONCLUSÃO}

A partir da análise das características dos atendimentos a vítimas de trauma, admitidas via transporte aéreo, foi possível identificar que as vítimas de trauma, admitidas via transporte aéreo, eram em sua maioria graves e demandaram atendimento imediato e especializado, incluindo propedêuticas e terapêuticas de alta complexidade. Mais da metade da população era do sexo masculino e da faixa etária economicamente ativa. A colisão automobilística foi o principal mecanismo de trauma, a maioria necessitou realizar exames de imagem e tratamento cirúrgico das lesões. A mediana do tempo de internação foi de sete dias. Mais da 
metade recebeu alta para o domicílio, porém, houve uma taxa de óbito elevada.

Conhecer o perfil e as condições de admissão e tratamento desses pacientes contribuem para repensar o preparo da equipe de saúde, a melhoria da articulação entre as unidades e serviços, o estabelecimento de fluxos de referência e contrarreferência, o investimento em educação permanente e continuada, além de melhor dispor os recursos intra e extrahospitalares, como o próprio transporte aéreo, a fim de tornar o atendimento mais ágil e eficaz, reduzindo custos e incapacidades. Os resultados deste estudo também podem embasar o aprimoramento de medidas preventivas ao trauma, fortalecendo a política estadual de atenção às causas externas e linha de cuidado traumatológica, contribuindo para o controle desse evento.

\section{REFERÊNCIAS}

1 - Institute for Health Metrics and Evaluation. GBD Compare: both sexes, all ages. Washington: National Institutes of Health; 2017 [citado em 28 mar 2020]. Disponivel em: https://vizhub.healthdata.org/gbd-compare/

2 - CID-10: International Statistical Classification of Diseases and Related Health Problems. DATASUS. Brasília: Ministério da Saúde; 2017 [citado em 28 mar 2020]. Disponível em: http://www.datasus.gov.br/cid10/V2008/WebHel p/cid10.htm

3 -DATASUS: sistema de informações sobre mortalidade. Brasil: Ministério da Saúde; 2017 [citado em 28 mar 2020]. Disponível em: http://tabnet.datasus.gov.br/cgi/tabcgi.exe?sim/c nv/ext10uf.def

4 - Alvarez BD, Razente DM, Lacerda DAM, Lother NS, Von-Bahten LC, Stahlschmidt CM. Avaliação do escore de trauma revisado em 200 vítimas de trauma com mecanismos diferentes. Rev Col Bras Cir. 2016;43(5):334-40. DOI: 10.1590/0100$\underline{69912016005010}$

5 - Silva RM, Costa HGF, Souza DMOR, Korinfsky JP, Silva TFA, Schwingel, P. A. Caracterização do atendimento às vítimas de acidentes de trânsito em um serviço público de emergência. Rev Enferm UFPE 2017;11(9):3650-61. DOI: 10.5205/reuol.10620-94529-1-

SM.1109sup201719
6 - Silveira ES, O'Dwyer G. Centro de trauma: Modelo alternativo de atendimento às causas externas no estado do Rio de Janeiro. Saúde Debate. 2017;41(112):243-54. DOI: 10.1590/01031104201711220

7 - Moraes DC, Brey C, Pizzolato AC, Caveião C, Sarquis LMM. Aplicação dos princípios do Prehospital Trauma Life Support. Cogitare Enferm. 2016;21(2):1-9. DOI: 10.5380/ce.v21i2.44274

8 - Carvalho BC, Souza AM, Silva DP. Caracterização dos pacientes acometidos por doenças traumáticas: Uma revisão bibliográfica. In: Anais do 1ㅇ Congresso Internacional de Enfermagem, 2009; Tiradentes. Tiradentes: Universidade de Tiradentes; 2017. p. 1-4.

9 - Brown JB, Gestring ML, Guyette FX, Rosengart MR, Stassen NA, Forsythe RM, et al. Development and validation of the air medical prehospital triage score for helicopter transport of trauma patients. Ann Surg. 2016;264(2):378-85. DOI: 10.1097/SLA.0000000000001496

10 - Michaels D, Pham H, Puckett $Y$, Dissanaike S. Helicopter versus ground ambulance: Review of national database for outcomes in survival in transferred trauma patients in the USA. Trauma Surg Acute Care Open 2019;4(1):1-3. DOI: 10.1136/tsaco-2018-000211

11 - Field T, Homer J, Moura E, Brophy K, Emerton $C$, Guerrero $C B$, et al. Atendimento pré-hospitalar no trauma. 8nd ed. Burlington: Jones \& Barlett Learning; 2017.

12 - Blera MS, Ribas JLC. Atuação do enfermeiro no transporte aeromédico. Cad Saúde e Desenv. 2018 [citado em 28 mar 2020]; 13(7):5-19. Disponível em:

https://www.uninter.com/cadernosuninter/index. php/saude-e-desenvolvimento/article/view/1033

13 - Lacerda LS, Araújo ERM, Neta FLA. Transporte aeromédico no estado do Piauí: Perfil das ocorrências. Rev Prev Infec Saúde 2017;3(2):20-6. DOI: $\underline{10.26694 / \text { repis.v3i2.6453 }}$

14 - Corpo de Bombeiros de Minas Gerais. Brasil: CBMMG; 2019 [citado em 2 maio 2020]. Disponível em: $\quad$ http://www.bombeiros.mg.gov.br/ component/content/article/32-embm/74483cbmmg-boauberaba.html 
15 - Fundação Hospitalar de Minas Gerais. Brasil: FHEMIG; 2020 [citado em 28 maio 2020]. Disponível em: http://www.fhemig.mg.gov.br/atendimento/comp lexo-de-urgencia-e-emergencia/hospital-joao-xxiii

16 - Pinho FM, Amante LN, Salum NC, Silva R, Martins T. Guideline das ações no cuidado de enfermagem ao paciente adulto queimado. Rev Bras Queimaduras 2016 [citado em 28 mar 2020]; 15(1):13-23. Disponível em: http://rbqueimaduras.org.br/details/288/ptBR/guideline-das-acoes-no-cuidado-deenfermagem-ao-paciente-adulto-queimado

17 - Mackway-Jones K, Marsden J, Windle J. Sistema manchester de classificação de risco. 2nd ed. Belo Horizonte: Folium; 2017.

18 - Damiani D. Uso rotineiro do colar cervical no politraumatizado: Revisão crítica. Rev Soc Bras Clin Méd. 2017 [citado em 28 mar 2020]; 15(2):131-6. Disponível em: http://docs.bvsalud.org/biblioref/2017/11/87561 5/152 131-136.pdf

19 - Rotondo MF, Fildes J, Brasel KJ, Chapleau W, Merrick C, Peterson N, et al. Suporte avançado de vida no trauma. 9nd Chicago: American College of Surgeons; 2012.

20 - Silva, F, Boes AA, Lazzarini DD, Busana JA, Nascimento ERP, Jug W. Vítimas de trauma por acidente de moto atendidas em serviço móvel de urgência. Rev Enferm UFPI 2015;14(3):71-8. DOI: $\underline{10.26694 / \text { reufpi.v4i3.4406 }}$

21 - Travassos MCP, Silva ICB, Lima JPB, Fonseca $\mathrm{CM}$, Sachett JAG, Osis SL. Fatores associados ao óbito e à reposição volêmica em pacientes com trauma por lesões penetrantes. Rev Rene 2016;17(4):520-8. DOI: $\quad 10.15253 / 2175-$ $\underline{6783.2016000400012}$

22 - Schweitzer G, Nascimento ERP, Nascimento KC, Moreira AR, Amante LN, et al. Intervenções de emergência realizadas nas vítimas de trauma de um serviço aeromédico. Rev Bras Enferm. 2017;70(1):54-60. DOI: 10.1590/0034-7167-2016$\underline{0311}$

23 - Malachias I, Leles FAG, Pinto MAS. Plano Diretor de Regionalização da Saúde de Minas Gerais. Belo Horizonte: Secretaria de Estado de Saúde de Minas Gerais, 2010.
24 - Alves JCF, Fank A, Souza LP, Lima MG. O papel do enfermeiro na oxigenioterapia: revisão narrativa da literatura. J Health Biol Sci. 2018;6(2):22-4. DOI: 10.12662/23173076jhbs.v6i2.1242.p176-181.2018

25 - Brasil, Ministério da Saúde. Portaria no 10, de 3 de janeiro de 2017. Redefine as diretrizes de modelo assistencial e financiamento de UPA $24 \mathrm{~h}$ de Pronto Atendimento como Componente da Rede de Atenção às Urgências, no âmbito do Sistema Único de Saúde. Diário Oficial da União $2017 . \quad$ Disponível em: https://bvsms.saude.gov.br/bvs/saudelegis/gm/20 17/prt0010 0301 2017.html

Nota: Este artigo é um produto do trabalho de conclusão de residência multiprofissional em saúde de Caio Henrique Macedo Camargos de Oliveira, na especialização em urgência e emergência, de um hospital público de grande porte.

Recebido em: 15/05/2020

Aprovado em: $11 / 10 / 2020$ 